\title{
A formação de acadêmicos de enfermagem quanto à percepção da dor em duas instituições de ensino superior*
}

\author{
Nursing students qualification as to pain perception in two universities.
}

Simone Regina A. de F. Barros ${ }^{1}$, Simey de Souza Leão Pereira², Adauto Almeida Neto ${ }^{3}$

* Recebido da Faculdade de Enfermagem de Arcoverde (FENFA), Arcoverde, PE, Brasil.

\section{RESUMO}

JUSTIFICATIVA E OBJETIVOS: A dor é um fenômeno universal, como sintoma ou doença, a dor é causa de procura pelo sistema de saúde tendo uma importante consideração na assistência de enfermagem. Dessa forma, o objetivo deste estudo foi analisar os conhecimentos adquiridos pelos acadêmicos de enfermagem acerca da dor e suas intervenções, em duas Instituições de Ensino Superior (IES).

MÉTODO: Estudo do tipo transversal de natureza quantitativa, utilizando como instrumento um questionário. A amostra estudada foi composta por 60 acadêmicos de enfermagem do último período da graduação. Realizado em duas Instituições de Ensino Superior Públicas, ambas localizadas no estado de Pernambuco, uma na região metropolitana do Recife e a outra na cidade de Arcoverde localizada na mesorregião do sertão pernambucano.

RESULTADOS: Da amostra analisada observou-se que $42 \%$ não concordaram em utilizar analgésicos em horários fixos para pacientes como risco de dor. $72 \%$ dizem não medicar o paciente se a dor for suportável. $93 \%$ não reconheceram a morfina, como primeira escolha para o

1. Enfermeira Sanitarista e Obstetra da Faculdade de Enfermagem de Arcoverde, PE, Brasil.

2. Professora Doutora em Tecnologias Energéticas e Nucleares, Centro de Ciências da Saúde da Faculdade de Enfermagem de Arcoverde, PE, Brasil.

3. Professor Mestre em Patologia Geral do Centro de Ciências da Saúde da Universidade Federal de Pernambuco, Recife, PE, Brasil.

Endereço para correspondência:

Simone Regina A. de F. Barros

Av. Jonas Camelo no 316 - Centro

56520-000 Buíque, PE.

Fone: (87) 3855-1311 ou (87) 9635-7484

E-mail: simoninhabarros2010@hotmail.com controle da dor nos pacientes que não correspondem à analgesia clássica. $80 \%$ não opinaram acerca das terapias alternativas. Os dados obtidos refletem um grau de confiabilidade estatística de $95 \%$.

CONCLUSÃO: Observou-se que a formação destes futuros profissionais apresenta limitações que podem contribuir para o sofrimento desnecessário dos pacientes que sofrem de dor.

Descritores: Aferição da dor, Estudo da dor, Formação do enfermeiro.

\section{SUMMARY}

BACKGROUND AND OBJECTIVES: Pain is a universal fact. As symptom or disease, pain leads to the health system assistance with important considerations in nursing assistance. So, this study aimed at analyzing knowledge acquired by nursing students about pain and its interventions, in two universities.

METHOD: This was a transversal, quantitative study, using a questionnaire. Sample was made up of 60 nursing students in the last graduation year, and was carried out in two public universities of the state of Pernambuco, one in the metropolitan region of Recife and the other in the city of Arcoverde, located in the Pernambuco's hinterland mid-region.

RESULTS: It has been observed that $42 \%$ disagreed with the use of analgesic drugs in fixed times for patients at risk of pain; $72 \%$ said they do not medicate patients if pain is bearable; $93 \%$ did not recognize morphine as the first choice for pain control of patients not responding to classic analgesia; $80 \%$ had no opinion about alternative therapies. Data obtained had a statistical reliability level of $95 \%$.

CONCLUSION: It has been observed that the qualification of these future professionals has limitations which may contribute to the unnecessary distress of patients with pain. Keywords: Nurse qualification, Pain measurement, Pain study. 


\section{INTRODUÇÃO}

A dor é uma queixa frequente que leva os pacientes à procura de um pronto-socorro, porém muitas vezes este sintoma não é bem abordado pela equipe que atua nos serviços, realizando avaliações imprecisas dos quadros de dor com subutilização do arsenal antiálgico disponível o que implica no controle inadequado da dor ${ }^{1}$.

Organizações como a Agência Americana de Pesquisa e Qualidade em Saúde Pública e a Sociedade Americana de Dor classificam a dor como um dos sinais vitais, já que deve ser avaliada e registrada com o mesmo rigor e seriedade da pressão arterial, frequência cardíaca, frequência respiratória e temperatura ${ }^{2}$.

As instituições formadoras de profissionais de saúde têm a responsabilidade de concretizar estes conceitos básicos sobre dor e terapêuticas para o controle mediante uma leitura interdisciplinar do fenômeno doloroso. Projetos encaminhados ao Ministério da Educação para que o tema dor fosse incluído nos currículos das faculdades da área da saúde estão ainda tramitando ${ }^{3}$. Sabe-se, portanto, que o ensino é o alicerce que delineia as formas de cuidar e merece devida atenção e análise reflexiva por parte dos formandos e dos formadores que integram o sistema universitário em nosso país.

A formação acadêmica do enfermeiro caracteriza-se pela abrangência que envolve o estudo de epidemiologia, microbiologia, imunologia, anatomia, fisiologia, patologia, farmacologia, nutrição, psicologia e psiquiatria, sociologia e saúde pública, entre outras disciplinas. Entretanto, a formação abrangente do enfermeiro permite-lhe importante versatilidade de ação e o coloca em posição única na equipe de saúde, pois o torna capaz de atuar considerando as perspectivas biológicas e psicossociais, podendo enfatizar diversas linhas de pensamento, dependendo das necessidades do paciente, das características do serviço e do profissional. De modo geral, pode-se inferir que a atuação do enfermeiro junto aos pacientes com dor pode ser dividida em três grupos: atendimento a pacientes em estados álgicos agudos, os com dores crônicas não oncológicas e com dores oncológicas ${ }^{4}$.

Diante desse contexto, o objetivo deste estudo foi analisar os conceitos adquiridos pelo acadêmico de enfermagem durante sua formação referente aos aspectos gerais e terapêuticos da dor, mediante o embasamento científico e prático, característico da graduação para assuntos e políticas nacionais e internacionais pertinentes à dor. Entretanto, é de grande relevância o conhecimento desta realidade acadêmica. Um diagnóstico adequado dos conceitos adquiridos pelos alunos de enfermagem durante a graduação pode ser a base para adequações necessárias nos currículos e disciplinas. Além disso, contribui na revisão do modelo atual de ensino frente às exigências do mercado de trabalho moderno que lança novos olhares sobre novos caminhos de fazer enfermagem.

\section{MÉTODO}

Após aprovação do Comitê de Ética em Pesquisa da Universidade de Pernambuco, com parecer $\mathrm{n}^{\mathrm{o}}$ 017/08, realizou-se este estudo transversal de natureza quantitativa e com abordagem descritiva. Realizado em duas Instituições de Ensino Superior (IES) Públicas ambas localizadas no estado de Pernambuco, uma na região metropolitana do Recife e a outra na cidade de Arcoverde, localizada na mesorregião do sertão pernambucano. A amostra estudada foi composta de 60 acadêmicos, os quais estavam cursando o último período do curso de bacharelado em enfermagem em ambas IES.

Com a finalidade de deixar os participantes cientes dos propósitos e da metodologia da pesquisa, foram explicados os objetivos almejados com a referida temática. No entanto, os participantes de acordo com seus interesses tiveram autonomia de escolher participar ou não da pesquisa.

Em atenção à resolução 196/96 do Conselho Nacional de Saúde que legisla sobre diretrizes e normas regulamentadoras de pesquisa envolvendo seres humanos os participantes que concordaram fazer parte da pesquisa assinaram um termo de consentimento denominado Termo de Consentimento Livre e Esclarecido.

Os dados foram coletados durante o primeiro semestre de 2008, por meio de um questionário contendo 10 questões abertas e fechadas.

Para a criação do banco de dados foi utilizada a planilha eletrônica do Programa Excel da Microsoft ${ }^{\circledR}$, o que possibilitou a organização dos dados em tabelas. Na análise estatística dos dados, foi utilizado o pacote estatístico SPSS (Statistiscal Package for the Social Sciences, 13.0). No entanto, alguns itens do questionário foram agrupados em aspectos relacionados à fisiologia da dor, avaliação da dor, terapêuticas farmacológicas/não farmacológicas pertinentes ao estudo da dor com o objetivo de facilitar a análise dos resultados. É válido salientar que os dados obtidos refletem um grau de confiabilidade estatística de 95\%.

\section{RESULTADOS}

Diante das informações obtidas a partir do instrumento utilizado e mediante o tratamento dos dados, foram selecionadas as assertivas de maior relevância para a 
compreensão dos questionamentos que direcionaram os objetivos deste estudo.

Observou-se que $56 \%$ da amostra analisada demonstraram ter recebido informações sobre o tema "dor", contudo $42 \%$ afirmam não ter recebido esse tipo de conhecimento específico e $2 \%$ preferiram abster-se de alguma resposta. Ainda observou-se que a grande maioria dos acadêmicos de enfermagem (95\%) não participaram de eventos, cursos e/ou minicursos sobre dor durante a sua formação.

É percebido que a grande maioria $(85 \%)$ concordaram que a dor pode ser caracterizada em termos de duração e doença, sendo que $12 \%$ não soube diferenciar a dor aguda da dor crônica quando questionados.

A tabela 1 mostra que a maioria dos entrevistados (38\%) não souberam opinar sobre os receptores específicos da dor. Enquanto 40\% não concordaram com os mecanismos periféricos da dor. $68 \%$ dos pesquisados afirmaram não conhecer a teoria do portão de controle da dor.

Acerca das medidas terapêuticas da dor é possível o uso de recursos farmacológicos e não farmacológicos. Diante deste contexto, a tabela 2 traz algumas considerações que são mais pertinentes.

Quanto ao aspecto medicar o paciente ou não, conforme se observou na tabela $2,72 \%$ dos alunos concordam em não medicar o paciente se a dor fosse suportável, sendo melhor investigar. Dentre os participantes da pesquisa $42 \%$ afirmaram que durante o tratamento com opioides as principais reações adversas são a taquicardia e agitação.
Observou-se que um número significativo de participantes $(42 \%)$ não concordaram em utilizar analgésicos em horários fixos para pacientes como risco de dor. Quanto ao critério combinação de fármacos, $48 \%$ dos entrevistados não concordaram e 18\% mostraram-se desconhecedores de tal administração. Observou-se que $40 \%$ não têm conhecimento sobre a ação inespecífica da maioria dos anti-inflamatórios. $48 \%$ dos entrevistados mostraram-se desconhecedores sobre o uso restrito de dipirona. Observou-se na pesquisa que $93 \%$ da amostra não reconhecem a morfina, como primeira escolha para o controle da dor, nos pacientes que não correspondem à analgesia clássica. $80 \%$ da amostra afirmaram não saber opinar sobre as terapias alternativas.

Um percentual de $52 \%$ dos participantes da pesquisa não apresentaram o diagnóstico para o caso clínico apresentado. Apenas 35\% apresentaram diagnósticos para o caso, embora apenas $13 \%$ dos diagnósticos foram de dor. Quanto às intervenções de enfermagem para o caso clínico foi apresentado um percentual de $31 \%$. Dessas apenas 58\% eram específicas para o caso clínico.

Observou-se ainda neste mesmo estudo que apenas $20 \%$ dos participantes da pesquisa classificaram os instrumentos de mensuração da dor. Desses, apenas 18,3\% mostraram-se capazes de utilizar a escala das faces de Wong Baker e a da escala da Mônica e Cebolinha.

Observou-se que em média $79 \%$ dos participantes da pesquisa apresentaram conhecimento incipiente com relação a todos os documentos oficiais, entidades, progra-

Tabela 1 - Distribuição das respostas relacionadas à fisiologia da dor. Faculdade de Enfermagem de Arcoverde-PE e Faculdade de Enfermagem Nossa Senhora das Graças, Recife-PE - 2008

\begin{tabular}{|c|c|c|c|c|}
\hline Aspectos Relacionados à Fisiologia da Dor & Concorda & Não Concorda & Não Sabe & NR \\
\hline $\begin{array}{l}\text { A dor geralmente é causada por estímulos térmicos, } \\
\text { químicos ou mecânicos. A energia desses estímulos é } \\
\text { convertida em energia elétrica (transdução). }\end{array}$ & $53 \%$ & $28 \%$ & $17 \%$ & $1 \%$ \\
\hline $\begin{array}{l}\text { Os receptores específicos para a dor estão localizados } \\
\text { nas terminações de fibras nervosas } A \delta \text { (nociceptivas) e } \\
\mathrm{C} \text { (nociceptores) de acordo com os mecanismos peri- } \\
\text { féricos da dor. }\end{array}$ & $20 \%$ & $40 \%$ & $38 \%$ & $2 \%$ \\
\hline $\begin{array}{l}\text { A teoria de controle da dor diz que há uma estrutura } \\
\text { complexa nos cornos dorsais da medula espinhal que } \\
\text { pode inibir a transmissão da dor; então, diz-se que o } \\
\text { portão será fechado. }\end{array}$ & $22 \%$ & $1 \%$ & $68 \%$ & $9 \%$ \\
\hline
\end{tabular}

$\mathrm{NR}=$ não responderam 
Tabela 2 - Distribuição das assertivas dos acadêmicos de enfermagem sobre os aspectos relacionados à terapêutica farmacológica e não farmacológica. Faculdade de Enfermagem de Arcoverde-PE e Faculdade de Enfermagem Nossa Senhora das Graças, Recife-PE - 2008

Aspectos Relacionados à Terapêutica da Dor: Farmacológica e Não Farmacológica

Se a dor for suportável é melhor não medicar e investigar a dor.

Durante o tratamento com opioides, toxicidade ou efeitos colaterais mais significativos que leva à necessidade de avaliação da enfermagem são a taquicardia e agitação.

É melhor utilizar analgésico em horários fixos para pacientes com risco de dor.

$\mathrm{Na}$ terapia farmacológica da dor é comum a administração combinada de vários medicamentos.

A morfina deve ser sempre a primeira escolha para a dor que não corresponde a analgésicos mais fracos.

Sem "dose limite" diária para seu uso.

A maioria dos anti-inflamatórios tem ação inespecífica, inibindo tanto a COX-1 como a COX-2 e seu uso crônico não causam repercussões renais e gastrintestinais.

A dipirona teve seu uso restrito devido ao risco, embora pequeno, de causar agranulocitose irreversível.

Só é terapeuta alternativo/complementar (na área específica), mediante conclusão e aprovação em cursos reconhecidos em instituição de ensino, com carga horária mínima de 360 horas.

\section{Concorda Não Concorda Não Sabe NR}

$72 \%$

$25 \%$

$3 \%$

$42 \%$

$33 \%$

$25 \%$

$\begin{array}{llll}50 \% & 42 \% & 8 \% & \\ 32 \% & 48 \% & 18 \% & 1 \% \\ 2 \% & 93 \% & 5 \% & \end{array}$

$5 \%$

$55 \%$

$40 \%$

$28 \%$

$23 \%$

$48 \%$

$10 \%$

$10 \%$

$80 \%$

$\mathrm{NR}=$ não responderam

mas de educação continuada e políticas públicas para o estudo/alívio da dor.

Os participantes da pesquisa ainda reconheceram que a sua formação para lidar extramuros da sala de aula no processo álgico não compreendem o desempenho esperado na formação de um profissional de enfermagem. Sendo que $50 \%$ afirmam ter conhecimento regular e $33 \%$ afirmam ter conhecimento insuficiente.

\section{DISCUSSÃO}

O desenvolvimento do tema dor de forma estanque e independente, sem elos necessários à compreensão clínica, dificulta o entendimento e resulta na formação de profissionais sem visão integrada do que seja a dor. Os da- dos apresentados nesta pesquisa recaem sobre o fato de os conteúdos sobre dor não terem sido abordados como temas principais e sim como conceitos complementares em diversas disciplinas ${ }^{10}$.

É de conhecimento público que o ensino na área da saúde e, mais especificamente, no curso de graduação em enfermagem, deve propiciar aquisição de conhecimentos e mudanças comportamentais, sem perder de vista a vinculação entre teoria e prática. Neste estudo demonstrou-se que $12 \%$ da população estudada não souberam diferenciar a dor aguda da dor crônica quando questionados.

A dor aguda produz uma série de respostas dentre elas: alterações ventilatórias e circulatórias, afetando também a função gastrintestinal, urinária e musculoesquelética. Sendo comum o paciente apresentar aumento da fre- 
quência respiratória, do volume sistólico (elevação da pressão arterial), diminuição do tônus gastrintestinal, retenção urinária e aumento das secreções neuroendócrinas (estresse). Além disso, as reações de ansiedade, apreensão e tremor, induzem respostas psicológicas, fisiológicas e condutas que caracterizam a experiência multidimensional da dor ${ }^{5}$.

Para o profissional da área de saúde é extremamente importante o conhecimento de forma integral da fisiologia da dor. Conforme os dados apresentados na tabela 2 um percentual significativo de acadêmicos não demonstraram conhecimento acerca da fisiologia da dor. $\mathrm{O}$ primeiro passo na sequência dos eventos que originam o fenômeno doloroso é a transformação dos estímulos agressivos em potenciais de ação que, das fibras nervosas periféricas, os quais são transferidos para o sistema nervoso central, podendo ser traduzidos de estímulos de natureza térmica, química ou mecânica, em estímulo elétrico, para ser interpretado no córtex cerebral como dor ${ }^{6}$. Após a agressão tecidual há liberação de neurotransmissores, como substância $\mathrm{P}$, somatostatina, peptídeo geneticamente relacionado com a calcitonina, neurocinina-A, glutamato e aspartato. Essas substâncias estão relacionadas com a ativação de potenciais pós-sinápticos excitatórios e dos receptores N-Metil-D-Aspartato (NMDA) e não $\mathrm{NMDA}^{7}$. Sendo de suma importância o seu conhecimento para o entendimento da mesma.

Os receptores específicos para a dor estão localizados nas terminações de fibras nervosas A $\delta$ (fibras delta-nociceptivas) e C (fibras amielinicas-nociceptores) e, quando ativados, sofrem alterações na sua membrana, permitindo a deflagração de potenciais de ação. As fibras $\mathrm{A} \delta$ são mielinizadas e as fibras $\mathrm{C}$ não são mielinizadas $\mathrm{e}$ possuem a capacidade de transmitir estímulos dolorosos em diferentes velocidades. As fibras $\mathrm{A} \delta$, em função da presença da bainha de mielina, transmitem o estímulo doloroso de forma rápida, enquanto as fibras $\mathrm{C}$ são responsáveis pela transmissão lenta da dor ${ }^{6}$.

A teoria do portão de controle da dor foi a primeira a sugerir que além da sensação física a dor tem componentes emocionais e cognitivos obrigatórios. No entanto, a teoria sugere que impulsos de dor se efetivam, quando o portão está aberto e são bloqueados quando o portão é fechado. No entanto, fechar o portão constitui a base das intervenções para o alívio da dor ${ }^{8}$. Observou-se, portanto, nesta pesquisa que $68 \%$ dos participantes não souberam opinar acerca dessa teoria.

Outro aspecto a ser avaliado nesta pesquisa foi quanto aos métodos utilizados para mensurar a percepção/sensação da dor. Vários métodos têm sido utilizados para mensurar a percepção/sensação de dor. Os instrumentos unidimensionais são designados para quantificar apenas a severidade ou a intensidade da dor e têm sido usados frequentemente em hospitais e/ou clínicas para se obter informações rápidas, não invasivas e válidas sobre a dor e a analgesia. São exemplos: a escala visual numérica (EVN), a escala analógica visual (EAV), escala verbal descritiva (EVD), escala das faces, escala da Mônica e Cebolinha e escala de Oucher dentre outros. Sendo as duas últimas utilizadas para mensuração da dor em crianças 9

Observou-se também neste estudo que apenas $20 \%$ dos participantes da pesquisa classificaram os instrumentos de mensuração da dor. Desses, apenas 18,3\% mostraram-se capazes de utilizar a escala das faces de Wong Baker e a da escala da Mônica e Cebolinha. A mensuração da dor é extremamente importante no ambiente clínico, pois se torna impossível manipular um problema desta natureza sem ter uma medida sobre a qual basear o tratamento ou a conduta terapêutica ${ }^{10}$.

Quanto aos aspectos relacionados à terapêutica farmacológica e não farmacológica, a pesquisa demonstrou que $72 \%$ dos alunos concordam em não medicar o paciente se a dor for suportável, sendo melhor investigar. Estes dados são discordantes da literatura, os estudos desenvolvidos na área de dor têm demonstrado que todo quadro de dor, se não for prevenido quando possível, ou tratado precocemente, pode levar ao aumento significativo da morbidade e mortalidade dos pacientes em todas as especialidades pesquisadas ${ }^{11}$. Como efeitos colaterais, os analgésicos opioides podem provocar sedação, náusea, vômitos, prurido, retenção urinária e constipação intestinal numa relação dose-dependente. Como também as altas doses de opioides podem levar não só à depressão respiratória, mas apneia, colapso circulatório e coma seguido de morte ${ }^{12}$. A analgesia com dose fixa tem como o objetivo o controle da dor ${ }^{11}$.

A administração combinada de fármacos tem se tornado uma realidade, como também, uma necessidade cada vez maior no ambiente clínico, contudo $48 \%$ dos entrevistados não concordaram e 18\% mostraram-se desconhecedores de tal administração.

Conforme se observou na pesquisa $93 \%$ da amostra não reconhecem a morfina, como primeira escolha. A morfina é um alcaloide natural da papoula, fármaco de escolha do tratamento da dor oncológica grave e seu uso é sem "dose limite" diária. Seu amplo uso deve-se à variedade em formulações, à facilidade na titulação da dose e a meia-vida curta. Essa também é a recomendação da Organização Mundial de Saúde (OMS). Em um levantamento da OMS realizado nos 10 países desenvolvidos 
que mais consomem opioides - principalmente a morfina - verificou-se, a partir de registros consistentes, que a morfina e outros opioides foram utilizados em até $90 \%$ das vezes para alívio da dor causada por doença não neoplásica e que houve aumento significativo do consumo no período de 1976 a 1992, mostrando variação desse incremento de $198 \%$ a $3.318 \%$ por país ${ }^{11}$.

Atualmente se conhecem dois tipos de COX. A COX-1 presente na maioria dos tecidos está relacionada à função renal (balanço de água e sódio), à agregação plaquetária e à proteção da mucosa gástrica. A COX-2 normalmente presente no sistema nervoso central e no aparelho urogenital, é induzida durante o processo inflamatório se expressando nos macrófagos e outras células dos tecidos inflamados. Portanto, a maior parte dos efeitos colaterais dos anti-inflamatórios não esteroides (AINES) estão relacionados à utilização AINES não seletivos e de ação na COX-1, principalmente irritação gastrintestinal e alteração da hemostasia. Observou-se, portanto que 40\% dos pesquisados não têm conhecimento sobre a ação inespecífica da maioria dos anti-inflamatórios ${ }^{12}$.

Apesar de a dipirona ser um fármaco extremamente popular e utilizado a nível terapêutico em larga escala, 48\% dos entrevistados mostraram-se desconhecedores do seu uso restrito ao risco, embora pequeno, de causar agranulocitose irreversível. Apesar de ter sido banida dos Estados Unidos em 1979 devido à associação com agranulocitose fatal, a dipirona continua ali sendo usada por imigrantes latinos, dando origem a casos de neutropenia ou leucopenia e grave infecção associada, como mostra um levantamento realizado com 113 pacientes de fala espanhola ou portuguesa durante 15 dias. Nele se aponta que o fármaco era usado por $35 \%$ deles, $20 \%$ dos quais o tinham em casa e $25 \%$ o haviam comprado nos Estados Unidos. De maneira geral, a frequência de agranulocitose é baixa, com variação de dados entre diferentes autores. Descreveu-se predisposição genética como fator presente na agranulocitose induzida por dipirona. Outros efeitos adversos também têm sido descritos. Estudo de casos e controles conduzido no Brasil encontrou associação entre aumento do risco de tumor de Wilms em crianças cujas mães usaram o fármaco durante a gestação ${ }^{13}$.

O exercício profissional requer legislações para o seu bom andamento, contudo $80 \%$ da amostra entrevistada afirmaram não saber opinar a despeito das Terapias Alternativas. No entanto a Resolução do COFEN 197/97 estabelece sobre as Terapias Alternativas como especialidades e/ou qualificação do profissional de enfermagem. Há que salientar que a enfermagem exerce papel fundamental nesse contexto já que é ela quem está em contato direto e mais profundo com a população, tendo a oportunidade de educá-la e esclarecê-la quanto ao uso (benéfico ou não) dessas técnicas, seja em hospitais, em centros de saúde ou junto à comunidade ${ }^{14}$.

Constatou-se que $66,1 \%$ dos enfermeiros docentes em quatro instituições de ensino de graduação em Enfermagem da cidade de São Paulo fazem uso das Terapias Alternativas/Complementares, embora, dos que fazem uso, apenas $58,9 \%$ falam desse assunto com seus alunos ${ }^{14}$.

Considerando que apenas $13 \%$ dos participantes da pesquisa apresentaram o diagnóstico para o caso clínico, é importante considerar que a credibilidade sobre a prática de enfermagem repousa principalmente sobre as atividades de enfermagem resultantes de diagnósticos. Pois é com a identificação do diagnóstico que surge concomitantemente ao questionamento sobre a seleção de prescrições para o caso. A abrangência dos diagnósticos de enfermagem é vasta, com grandes vantagens de clarear os fenômenos, os problemas de enfermagem e, sem duvida, ajudam a organizar a delimitar a coleta de dados. $\mathrm{O}$ levantamento de dados é o alicerce no qual se fundamenta o cuidado de enfermagem ${ }^{15}$.

\section{CONSIDERAÇÕES FINAIS}

Os resultados permitem concluir que a formação destes futuros profissionais apresenta limitações que podem contribuir para o sofrimento desnecessário e diminuição da qualidade de vida dos pacientes que sofrem de dor. Embora uma pessoa consiga sobreviver com dor, ela interfere no seu bem estar, nas relações sociais e familiares, no desempenho do seu trabalho, influenciando assim na sua qualidade de vida.

Acredita-se que as instituições formadoras desses profissionais de enfermagem não parecem estar preparando seus futuros enfermeiros para lidar com a dor na área clínica. $\mathrm{O}$ objetivo fundamental é que o aluno entenda o fenômeno doloroso, agudo ou crônico, e suas repercussões biopsicossociais e sensibilize-se para importância dos cuidados que devem ser prestados no processo álgico.

Em se tratando de um campo de saberes e práticas em construção, a articulação das disciplinas da graduação deve ser mais estreita, principalmente a despeito do tema dor permitindo maior impacto na formação e consequentemente nas práticas de enfermagem.

A responsabilidade atribuída ao enfermeiro para lidar na dor e os inúmeros aspectos que dele dependem para uma boa assistência talvez ajudem a entender o motivo da grande valorização e preocupação da precisão técnica e seu embasamento em conhecimentos científicos. 
Vale ressaltar que o desconhecimento dos alunos sobre o respaldo legal de especializações (terapias alternativas) para o enfermeiro chama a atenção, pois, se os acadêmicos não sabem sobre seus direitos, talvez também não estejam plenamente conscientes sobre seus deveres profissionais.

A formação de um profissional mais seguro e consciente reflete no desenvolvimento de uma assistência diferenciada ao paciente. Possibilita ao cuidador desempenhar suas funções de forma cautelosa e reflexiva, evitando a transgressão de valores e convicções, permitindo o estabelecimento de relacionamentos interpessoais mais efetivos entre profissional e paciente.

\section{REFERÊNCIAS}

1. Ponte STD, Machado A, Dutra APG, et al. Dor como queixa principal no serviço de pronto-atendimento do hospital municipal de São Pedro do Sul- RS. Rev Dor 2008;9(4):1345-9.

2. Sousa FAEF. Dor: o quinto sinal vital. Rev Latino-Am Enfermagem 2002;10(3):446-7.

3. Newton B. Manejo da dor no Brasil - Ação Decisiva da SBED. Rev Dor 2005;6(4):645.

4. Pimenta CAM. Proposta de conteúdo mínimo sobre dor e cuidados paliativos nos cursos de graduação da área de saúde. Revista Simbidor 2005;2(1):7.

5. Braun Filho JB, Braun L. Dor aguda. Rev Dor 2004;1(2):3-14

6. Rocha AP, Kraychete DC, Lemonica L, et al. Pain: current aspects on peripheral and central sensitization. Rev Bras Anestesiol 2007; 57(1):94-105.

7. Strong J, Ashton R, Chant D. The measurement of atti- tudes towards and beliefs about pain. Pain 2002;48(2): 227-36.

8. Santana JM, Lauretti GR. Possíveis mecanismos de ação da estimulação elétrica nervosa transcutânea no controle da dor. Rev Dor 2006;7(1):716-28.

9. Rigotti MA, Ferreira AM. Intervenções de enfermagem ao paciente com dor. Arq Ciênc Saúde 2005; 12(1):4-50.

10. Sereza TW, Dellaroza MSG. O que está sendo aprendido a respeito da dor na UEL? Semina: Ciências Biológicas e da Saúde 2004;24(1):55-66.

11. Moreno JR. A teoria moderna da dor e suas consequências práticas. Prática Hospitalar 2004;35(6):1.

12. Desjardins PJ, Grossman EH, Kuss ME, et al. The injectable cyclooxygenase-2-specific inhibitor parecoxib sodium has analgesic efficacy when administered preoperatively. Anesth Analg 2001;93(3):721-7.

13. Wannmacher L, Ferreira MBC. Febre: mitos que determinam condutas. Organização Pan-Americana da Saúde; Organização Mundial da Saúde - Brasil. Brasília - DF 2004;8(1):1-14.

14. Trovo MM, da Silva MJ, Leão ER. Alternative/ complementary therapies in public and private education: analysis of knowledge among nursing students. Rev Lat Am Enfermagem 2003;11(4):483-9.

15. Pimenta CA, da Cruz DA, de Araujo TL, et. al. The teaching of patient evaluation: the delineation of the content of nursing diagnosis. Rev Lat Am Enfermagem 1993;1(2):69-76.

Apresentado em 05 de janeiro de 2011.

Aceito para publicação em 08 de junho de 2011. 